\title{
Comparison of Ultrasonic Methods for Detecting Defects in Unidirectional Composite Material
}

\author{
Vanessa Vieira Gonçalves ${ }^{a}$ (1), Daniel Marcus Giglioli de Oliveira ${ }^{*}$ * (1), \\ Auteliano Antunes dos Santos Junior ${ }^{a}$ (i) \\ ${ }^{a}$ Universidade Estadual de Campinas (Unicamp), Campinas, SP, Brasil.
}

Received: June 29, 2021; Revised: August 23, 2021; Accepted September 26, 2021

\begin{abstract}
Non-destructive tests are of pivotal importance in the industry, as they contribute to the decreasing of maintenance costs, reducing downtime and accidents. Ultrasound is a non-destructive method that can be used with several arrangements to identify faults in engineering materials. This work evaluated the use of three ultrasonic methods applied in FRP composites to detect manufacturing defects. The critically refracted longitudinal wave (LCR), the B-Scan images, and the signal-to-noise ratio of TFM (Total Focusing Method) images were compared to detect the delamination and the fiber waviness in unidirectional composite of carbon/epoxy. Rectangular samples without defects and with defects were used in the tests. To obtain the delamination in the samples, pieces of Teflon were placed between layers and for the samples with waviness, a silica sphere was used. The results showed that LCR wave is not recommended to detect these kinds of defects, the B-Scan image is efficient in detecting delamination, and the SNR variation of TFM images allows to identify the waviness and delamination in unidirectional FRP composites.
\end{abstract}

Keywords: Non-destructive inspection, ultrasound, unidirectional composite, LCR wave, phased array.

\section{Introduction}

Among the materials that are used to make mechanical components, the composites are widely used in the aerospace industry, with aircraft having up to $80 \%$ of their structural volume made of them, such as the Boing $787^{1}$. Besides, high speed trains, wind energy equipment, oil and gas, defense, naval, automotive, and construction industries widely employ these materials ${ }^{2-4}$. Their advantages are tailorable properties, high strength, low density, corrosion resistance, and fatigue strength ${ }^{5,6}$. Unlike with metals, the damages appear prematurely ${ }^{7}$, but they usually propagate in a stable way. This apparent contradiction makes them advantageous when compared to metals, particularly for parts under variable loads, subject to fatigue. The reason is the complex and interactive relationship between fibers, damage, and residual stresses that blocks the spread of the damage ${ }^{8}$. A critical issue with composites is their vulnerability to low energy/speed impacts, which can cause considerable damage to the interior of the parts without anomalies on the impacted surfaces. Sometimes, the damage is imperceptible in visual inspections ${ }^{9,10}$. This is even more critical with structures and covers in equipment where life risks are involved.

Because of the interest in the use of composite materials in primary structures, a current aim is to reduce the defects induced along the manufacturing process. Such defects are undesirable, but they can be tolerable in some extension in secondary structures. In case of fiber-reinforced-polymer (FRP), defects can occur in fiber, matrix or in the interface.

*e-mail: dmoliveira@fem.unicamp.br
The main ones are delamination, fiber break, crack in the matrix, debonding of the fiber-matrix interface, waviness, porosity, among others ${ }^{11,12}$.

The main concern with the presence of defects in the composites is related to the change in the mechanical properties and nonlinear behavior that they can cause in the parts ${ }^{10}$. A common defect is the fiber waviness, in-plane and out-of-plane, which can arise in the prepregs molding process, due to gaps unfilled or partially filled between the layers. That defect can result in areas rich in resins. Moreover, the waviness can also be the result of thermoelastic distortions along the thickness or arises when the composite is used to manufacture curved components ${ }^{13}$.

Fiber waviness is classified as one of the main causes of failure in service. It is directly related with the deterioration of the mechanical properties of the components. When in-plane, it has a more severe and predominant effect on the mechanical performance than the out-of-plane ${ }^{14,15}$, though compressive strength is more sensitive to waviness out-of-plane ${ }^{16}$. This defect also causes a reduction in the natural frequency of the laminate ${ }^{17}$, affects the thermal properties ${ }^{18}$, the mechanical resistance ${ }^{16,19}$ and the fatigue strength ${ }^{20,21}$.

Another common defect in composites is delamination, expressed as a crack at the interface between adjacent layers of the laminated composite ${ }^{22}$. In delamination, high interlaminar stresses cause gaps along the part thickness. Delamination can arise during the service life, due to debris impact, tool drop during maintenance, hole drilling ${ }^{23}$, and also from the geometric configuration of the part, such as in straight free edges, curved free edges, external ply drop, corner, skin stiffener interactions, solid-sandwich transitions, 
and internal ply drops ${ }^{22}$. The presence of delamination can interfere with the mechanical behavior of the part. Studies have already shown that it reduces the stiffness of structures and decreases its natural frequency ${ }^{24}$, in addition to affect the material compressive strength ${ }^{25}$.

Resuming, both delamination and fiber waviness affect the mechanical behavior of the laminated composite, which justifies the need to identify and monitor the presence of these defects in components. For this aim, reliable and efficient inspection techniques must be applied.

There are studies addressing the application of different methods to detect delamination and fiber waviness, such as X- Ray ${ }^{15}$, eddy current ${ }^{26,27}$, thermography ${ }^{28}$, and guided waves $^{29}$. The use of a pulsed terahertz electromagnetic inspection method has also proved to be viable for detecting fiber waviness, generating multiple reflections as a response in the fold region with fiber waving for laminated glass composite materials ${ }^{30}$. This method has the advantage of having high-resolution and works without contact. Besides, it is non-ionizing and safer. Dong et al. ${ }^{31}$ compared terahertz imaging and ultrasonic $\mathrm{C}$-scans for detecting delamination in glass fiber samples. However, for carbon fibers, its conductivity only makes it possible to detect defects close to the surface.

Considering that $27 \%$ of the life cycle cost of a composite material is spent on inspection and repairs ${ }^{32}$, it is clear that the advantages of this material can only be fully exploited with the development of cheaper and more reliable monitoring and inspection techniques ${ }^{33}$.

Among the non-destructive evaluation (NDE) methods, the ultrasound is widely used for its low cost and easy handling. In addition, it can be applied with different techniques such as pulse echo or direct transmission, and different types of waves, such as longitudinal, transversal, and subsurface waves. The analysis can employ the amplitude of the signal, the time-of-flight (TOF), B-Scan image, and signal-to-noise ratio (SNR) of an image obtained using Total Focusing Method (TFM). These characteristics and the flexibility make the ultrasonic method quite versatile. Furthermore, numerous information can be provided by the techniques, such as defect detection, residual stresses, elastic properties, and so on ${ }^{34,35}$.

Several methods are being compared to ultrasound to detect defects in composites, as X-ray ${ }^{36}$, thermography ${ }^{37}$ and others . For the ultrasonic method, some studies evaluate the surface and subsurface wave propagation to identify these imperfections ${ }^{32,33,38}$. Phased array (PA) application is a current field of study, because it allows to generate real-time images of the internal structure ${ }^{39}$. Images from PA systems can also be recorded and analyzed for post-processing ${ }^{40}$.

In the case of composites, their anisotropic characteristics interfere with the propagation of the wave, which is also dependent on the direction of the fiber, defects, and the stresses inside the material, among others. As a result, the influences in the application of the ultrasonic method must be evaluated.

Feng et al. $^{41}$ simulated and evaluated the interaction of the S0 and A0 modes of Lamb waves in a laminated composite with delamination, verifying that the strongest mode conversions occur when the wave meets the edges of a delamination. Besides, S0 mode suffers more influence of delamination, generating $\mathrm{S} 0$ and $\mathrm{A} 0$ waves at the end of the laminate, with different velocities. In this condition, $\mathrm{S} 0$ is greater than A0. This difference makes it possible to estimate the location of the delamination.

The presence of fiber waviness causes deviation of the ultrasonic beam. When using oblique incidence angles on this kind of defect, the response is asymmetric and there is a change in the position of maximum energy. Combining with the double scanning technique, it is possible to determine the relative displacement of this energy. Zardan et al. ${ }^{42}$ performed an experimental analysis of this phenomenon and detected it even in thick components, but the method required access to both sides of the component.

After unsuccessful attempts to use focused C-Scan and a pair of transducers in transmission mode to detect defects in coarse samples, due to high refraction of the beams, Wooh and Daniel ${ }^{43}$ applied a paintbrush transducer with a special set-up to perform the task. Their studies have shown that longitudinal waves are reflected in the defects and that transverse waves showed a particular intensity pattern in the presence of fiber waviness. The analysis was based on elastodynamic ray theory, which is valid for high frequencies.

For delamination, a qualitative characterization is possible with lamb waves, in which the velocity of propagation, energy, and wave form are related to the extension of the delamination. The analysis is possible by combining the Fast Fourier Transform (FFT), Hilbert transform (HT), and the continuous wavelet transform $(\mathrm{CWT})^{44}$.

Alternative ways of applying PAS were studied by Kappatos et al. ${ }^{45}$. According to their work, changes in the characteristics of the probe, such as inclination, elevation, number of elements in the scan, in addition to the positioning distance, can interfere with the image resolution.

Employing ultrasound for composite inspection is not trivial because of the complexity of wave reflections due to inhomogeneity of the material. Zhang et al. ${ }^{46}$ showed the waviness fiber, for example, can reduce the reflection of a defect by $50 \%$; thus, when using different frequencies and a signal post-processing to detect different coexisting defects, the B-Scan method is more reliable. FMC/TFM has also been under study; Larrañaga-Valsero et al. ${ }^{47}$ obtained better results with this method compared with immersion and conventional phased array to detect waviness out-of-plane applying a velocity correction approach in the TFM method, while Liu et al. ${ }^{48}$ used TFM a dynamic homogenization analysis of wave propagation in composite material and obtained better results with a frequency of $2 \mathrm{MHz}$. For them, when the sample thickness is smaller, higher frequencies shows to be more advantageous; thus, when detecting different defects with different sizes and layer thickness, approaches with different frequencies and time corrections looks to be the best alternative.

In line with the current state-of-art in the manufacturing defects detection, this work compared the efficient of three ultrasonic methods to detect delamination and fiber waviness in unidirectional composites of carbon fiber and epoxy resin. The method was tested using a subsurface wave, meaning the critically refracted longitudinal wave (LCR wave), B-Scan images, and the signal-noise ratio (SNR) of TFM images. The last two methods employed phased array systems (PAS). 


\section{Critically Refracted Longitudinal Wave (LCR wave)}

There are several types of waves that can be used for NDE such as Rayleigh waves, shear waves, longitudinal waves, and critically refracted longitudinal waves (LCR), among others. These waves are shown in Figure 1. Rayleigh waves are superficial waves; their particles move elliptically and suffer great attenuation in the direction of the thickness, being approximately $93 \%$ slower than shear waves in materials with a Poisson coefficient of $0.33^{49}$. Shear waves (S-Wave) are slower than the longitudinal (P-Wave) ones, with the particle moving in the transverse direction and the wave propagating in the longitudinal direction. In longitudinal and LCR waves, the particle and the wave move in the longitudinal direction. Bray et al. ${ }^{50}$ were the first group to study the sensitivity of various types of waves to stress in rails, demonstrating that the critically affected longitudinal waves (LCR) are the most sensitive.

When an incident wave hits the interface between a medium 1 and a medium 2, two sets of waves appear: reflected waves, that will remain in medium 1 , and refracted waves, that will propagate in medium 2. Obviously, in the case of NDE, the greatest interest is in the behavior of the refracted waves in medium 2 . The waves reflected in medium 1 (probe) are usually disregarded, but can be used as a calibration indicator ${ }^{50}$.

The set of refracted waves follows Snell's law, which describes the relationship between the propagation velocities of the incident and the refracted waves, according to Equation 1.

$$
\frac{\operatorname{Sin} \theta_{1}}{\operatorname{Sin} \theta_{2}}=\frac{V_{1}}{V_{2}},
$$

In Equation 1, $\Theta_{1}$ is the angle of the incident wave, $\Theta_{2}$ the angle of refraction, $V_{1}$ velocity of the incident wave, and $V_{2}$ is the velocity of the refracted wave. A consequence of Snell's law is that there are two critical angles: the first occurs when the longitudinal waves propagate at $90^{\circ}$ in medium 2 , and the second is when the shear waves propagate at $90^{\circ}$ in medium 2.
The critically refracted longitudinal waves are precisely the longitudinal waves that propagate at first critical angle, therefore they are parallel and close to the surface ${ }^{51}$. To generate the LCR wave, plastic wedges are used. To calculate the wedge angle, it is necessary to know the propagation velocity of the longitudinal wave in medium $1\left(V_{l}\right)$ and in medium $2\left(V_{2}\right)$. Considering $\Theta_{2}=90^{\circ}$ in Equation 1, it is possible to calculate $\Theta_{2}$, which will be the angle of fabrication of the wedges (probe), as can be seen in the detail of Figure 1.

The LCR wave has advantages in detecting defects near the surface, because its energy is concentrated up to $30^{\circ}$ from the surface $e^{51,52}$ and it travels up to two wavelengths. In addition, LCR waves are less sensitive to roughness of the material, because they are body waves, not surface waves. As an additional advantage, the method requires the access only to one surface of the component ${ }^{53}$, unlike through transmission techniques, which require access to two surfaces ${ }^{54}$.

\section{Phased Array System}

The ultrasonic array is generated with a probe formed by several piezoelectric elements (multi-elements) that can be geometrically arranged in a linear, matrix, or circular form. These elements are connected to a control unit that emits pulses following a delay law. The wave fronts of each element are subject to Huygens interference. Due to the delay law, it is possible to control the direction and depth of the emitted signal. When this delay law is applied, the probe is called phased array ${ }^{55}$, as shown in Figure 2.

Among the advantages of using a phased array system, the possibility of obtaining images in real-time is worth to mention. One of the methods generates B-Scans, which are $2 \mathrm{D}$ images of the cross section of the component, built by stacking A-Scan signals as a function of the time, for each position.

Another way to obtain images with the phased array is with the post-processing of signals by the method FMC (Full Matrix Capture). In this method, for the linear array probe, one element emits the pulse and all elements record the received signals, then the next element emits the pulse

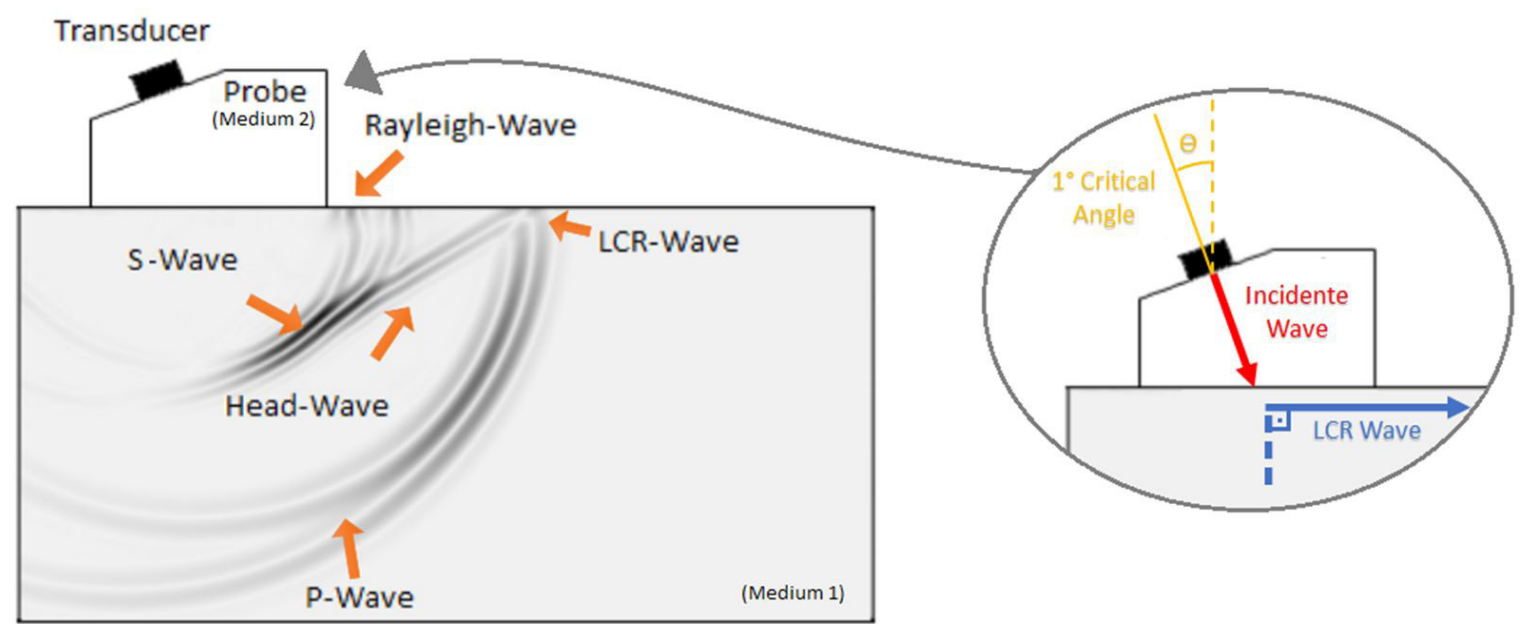

Figure 1. LCR wave generation. 
and all elements record the received signals. This process is repeated until all elements have emitted an ultrasonic pulse. The data obtained with the FMC method are applied to the image algorithm called TFM (Total Focusing Method) ${ }^{40}$. Using this method, the intensity of selected points on the images are obtained by Equation 2:

$$
\begin{aligned}
& I(x, z)=a b s\left|\sum_{i=1}^{n} \sum_{j=1}^{n} h_{i j}\left(t_{x, z}^{i j}\right)\right| \\
& t_{x, z}^{i j}=\frac{\sqrt{\left(x-x_{i}\right)^{2}+z^{2}}+\sqrt{\left(x-x_{j}\right)^{2}+z^{2}}}{c}
\end{aligned}
$$

In Equation 2, $I(x, z)$ is the intensity of the pixels, $h_{i j}\left(t_{x, z}^{i j}\right)$ is the amplitude of the pulse at the time it leaves the emitting element $i$ and arrives at the receiver element $j$. In Equation 3, $x_{i}$ and $x_{j}$ are the locations of the elements $i$ and $j$, and $\mathrm{c}$ is the velocity of the longitudinal wave.

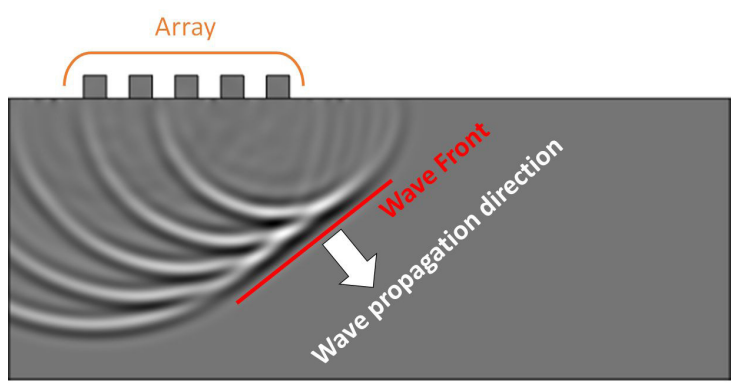

Figure 2. Wave front in a probe of phased array.

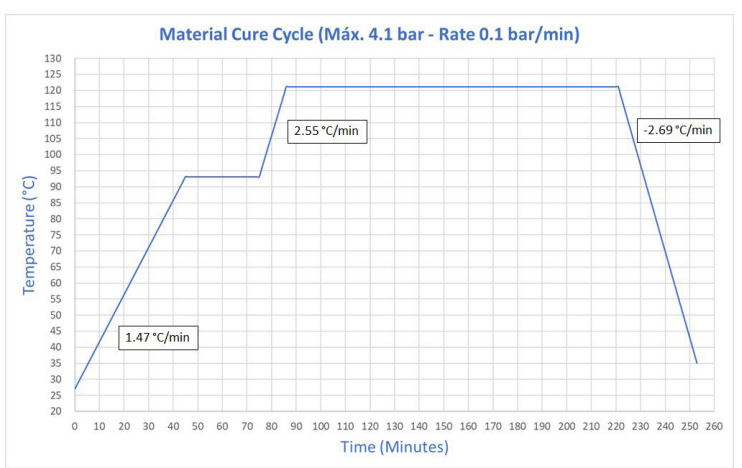

Figure 3. Prepreg material cure cycle.

\section{Materials and Methods}

To evaluate the selected techniques of ultrasound for detecting defects in unidirectional carbon/epoxy composite, specimens with prepreg carbon/epoxy SE 84LV Gurit ${ }^{\circledR}$ were manufactured. This prepreg was stored at $-18{ }^{\circ} \mathrm{C}$ before processing, to keep its properties. The lamination of samples took place in a white room (clean room) with stable temperature, humidity and controlled particulate rate. Due to the thickness, the specimens were manufactured in stages, involving a vacuum pre-compaction ( -81 bar) before the curing cycle process. The three initial cycles built 10 layers each and the last one 12 layers, totaling specimens with 42 layers. The vacuum bag was then taken to an autoclave, which followed the curing cycle shown in Figure 3.

At the end of the process described above, three groups of rectangular samples with dimensions $300 \times 40 \times 12.5 \mathrm{~mm}^{3}$ were obtained. The first group is composed of four samples with delamination (group T), the second of four samples with fiber waviness (group S), and the last set without any known defect (group CP), also of four samples. In the samples with delamination, a piece of Teflon $15 \times 10 \mathrm{~mm}^{2}$ was added between the layers 10 and 11 of the prepreg during the lamination process; a silica sphere was added between the layers 20 and 21 to produce the fiber waviness. Both the silica and the Teflon pieces are positioned centrally in the $\mathrm{x}-\mathrm{y}$ plane; the $\mathrm{z}$ axis is considered in the direction of the material thickness. The samples and the schematic of the defects are shown in Figure 4.

For the experiments with LCR waves, the measurement system consists of a pulser/receiver Ultratek USB UT-350, with a sampling rate of $50 \mathrm{MHz}$, connected to the computer by a USB cable. The data were processed and saved in a program made in Labview ${ }^{\circledR}$. Transducers of $2.25 \mathrm{MHz}$ were placed in wedges with a critical angle of approximately $15^{\circ}$. This angle was obtained by Snell's Law, Equation 1. For the propagation of the longitudinal wave at the first critical angle, the velocity of the wave in the wedge is $2300 \mathrm{~m} / \mathrm{s}$ and in the unidirectional composite in the fiber direction it is $9400 \mathrm{~m} / \mathrm{s}$. These wedges are fixed, and an aluminum bar was used to maintain a constant distance between them; the coupling between probe and sample was a conductive gel, Carbogel $\AA$. The measurement system is shown in the Figure 5.

For this first analysis, the samples were identified with markings in their surfaces. Lines were drawn, splitting their surfaces in three regions. The probe was then placed in each region, the emitter received the pulse, and the wave train at the receiver was recorded.

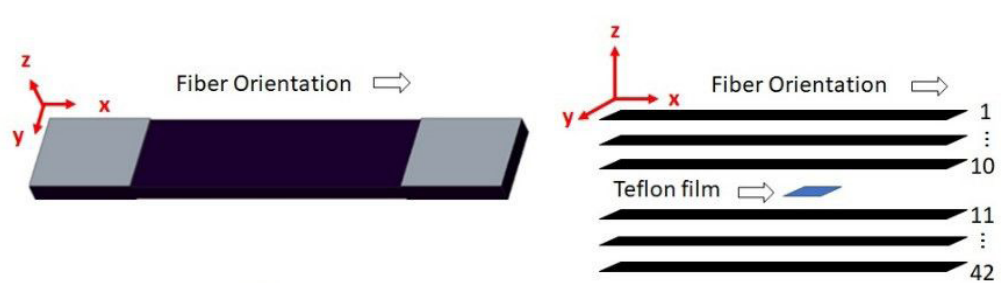

(a) (b)

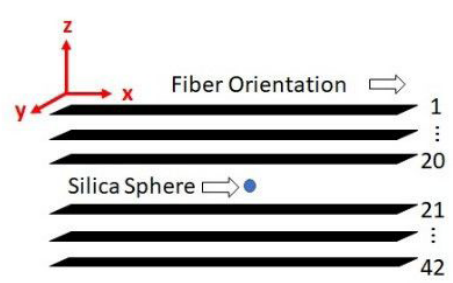

(c)

Figure 4. Sample scheme (a) rectangular sample; (b) Teflon between layers; (c) silica sphere between layers. 


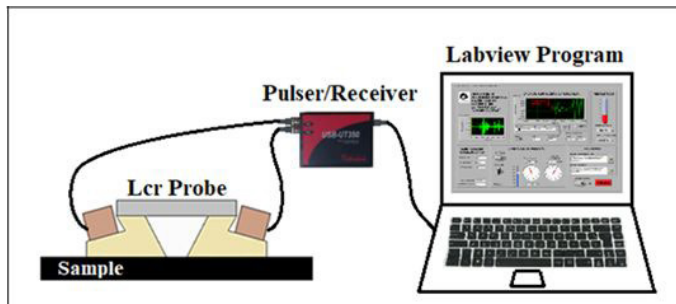

(a)

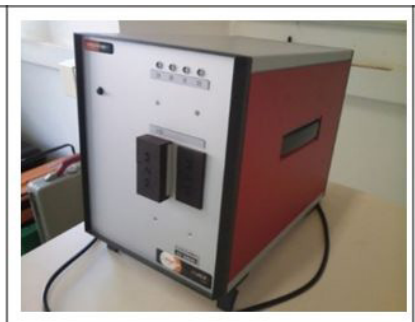

(b)

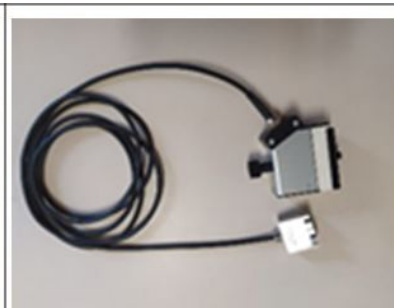

(c)

Figure 5. Measurement systems. (a) LCR wave equipment (out of scale) (b) PA system control unit (c) PA probe phased array.

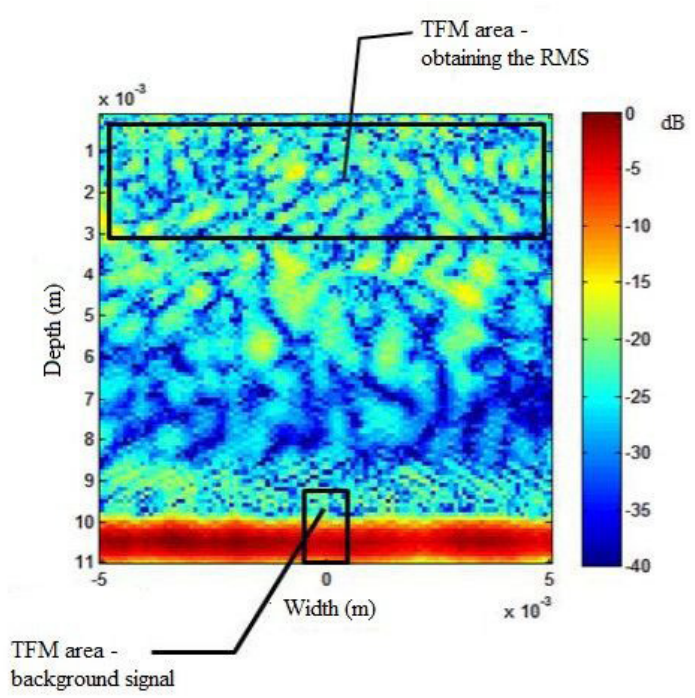

Figure 6. TFM image showing the main regions employed with the TFM method.

The B-Scan images were obtained with a phased array linear probe of 64 elements and $10 \mathrm{MHz}$. The probe was connected to a control unit M2M-MultiX++ (Figure 5) and the samples were engraved with their codes. Lines splitting their surfaces in four positions were drawn around and on the defect, where the images were recorded.

To obtain the signal-to-noise ratio (SNR) with the TFM image, the same PA system was used. For that, each sample surface was delimited in seven positions, then the FMC data were recorded with the phased array probe and used to construct the TFM image, shown in Figure 6. The value of SNR is obtained according to Equation 4.

$S N R=20 \log \left(\frac{I_{\text {peak }}}{I_{R M S}}\right)$

In Equation 4, $I_{\text {peak }}$ is the maximum intensity at the bottom of the sample and $I_{R M S}$ is the root mean square (RMS) of the intensity in the selected region of the TFM image.

For all techniques and measurements, the temperature of the sample was maintained at $23 \pm 1^{\circ} \mathrm{C}$.

\section{Results}

The results for the ultrasonic methods under evaluation are presented in this section. The LCR waves are evaluated by the value of the time-of-flight (TOF). Table 1 (samples without defects), Table 2 (samples with delamination) and Table 3 (samples with waviness) show the TOF values for the three positions in each sample and the standard deviation for each sample. The analysis with the TOF checked if it can be used to identify defects. Thus, when comparing the measured time between the positions of the same sample, it was expected to find significant differences in the time values. However, the results indicated in tables show a small variation in time between positions, which impedes the precise location of the defect.

The maximum average deviation for samples with delamination was $9 \mathrm{~ns}$ and in samples with fiber waviness was 12 ns. This variation does not contribute to identify the defect location.

The results obtained with the PA system are both by B-Scan images and SNR variation of TFM images. Figure 7, 8 and 9 show the B-Scan images obtained for samples without defects (called group CP), with fiber waviness (called group S) and delamination (called group $\mathrm{T}$ ), respectively. In some samples of group $\mathrm{S}$ is possible to identify a non-uniformity in the image that refers to the silica sphere, but it is not easy to identify the defect in all samples. There are rectangular marks indicating the presence of fiber waviness, but the reflections interfere with the accuracy of its depth. Figure 9 highlights the delamination identified in group $\mathrm{T}$. In this case, the images are clear, indicating the presence and location of the defect. The B-Scan method allowed the visualization of delamination in all samples from group $\mathrm{T}$, indicating a depth of $4 \mathrm{~mm}$, which coincides with the position of the Teflon pieces added to the laminates.

Figure 10 and Figure 11 show the results of the SNR variation in the TFM images along the samples with fiber waviness and with delamination, respectively. The values for each position in samples with defect are shown in the graphs along with values for samples without defects. Thus, the analysis of results is by comparison with values of the samples without defects (group CP).

The variation of SNR values in samples without defects is small and the fit curve obtained is approximately linear. The difference between the highest and lowest SNR values measured between positions of the same sample is $0.72 \mathrm{~dB}$. Figure 10 shows changes in SNR values in samples with fiber waviness, which indicate a difference of the highest and lowest value of $1.31 \mathrm{~dB}$. This difference was enough to identify the presence and location of defects, especially 
Table 1. Time of flight $(\mu \mathrm{s})$ of the LCR wave in samples without defects.

\begin{tabular}{ccccc}
\hline Sample & Position 1 $(\mu \mathrm{s})$ & Position 2 $(\mu \mathrm{s})$ & Position 3 $(\mu \mathrm{s})$ & Standard deviation $(\mu \mathrm{s})$ \\
\hline CP1 & 22.573 & 22.589 & 22.566 & 0.012 \\
\hline CP2 & 22.574 & 22.563 & 22.587 & 0.012 \\
\hline CP3 & 22.553 & 22.509 & 22.522 & 0.023 \\
\hline CP4 & 22.621 & 22.574 & 22.562 & 0.031 \\
\hline
\end{tabular}

Table 2. Time of flight ( $\mu$ s) of the LCR wave in samples with delamination.

\begin{tabular}{ccccc}
\hline Sample & Position $1(\mu \mathrm{s})$ & Position $2(\mu \mathrm{s})$ & Position 3 $(\mu \mathrm{s})$ & Standard deviation $(\mu \mathrm{s})$ \\
\hline T1 & 21.547 & 21.537 & 21.528 & 0.009 \\
\hline T2 & 21.574 & 21.572 & 21.563 & 0.006 \\
\hline T3 & 21.476 & 21.473 & 21.464 & 0.006 \\
\hline T4 & 21.574 & 21.578 & 21.588 & 0.007 \\
\hline
\end{tabular}

Table 3. Time of flight ( $\mu$ s) of the LCR wave in samples with fiber waviness.

\begin{tabular}{ccccc}
\hline Sample & Position $1(\mu \mathrm{s})$ & Position $2(\mu \mathrm{s})$ & Position 3 $(\mu \mathrm{s})$ & Standard deviation $(\mu \mathrm{s})$ \\
\hline S1 & 21.924 & 21.929 & 21.928 & 0.003 \\
\hline S2 & 22.008 & 21.997 & 22.007 & 0.006 \\
\hline S3 & 22.134 & 22.138 & 22.116 & 0.012 \\
\hline S4 & 22.121 & 22.137 & 22.124 & 0.008 \\
\hline
\end{tabular}
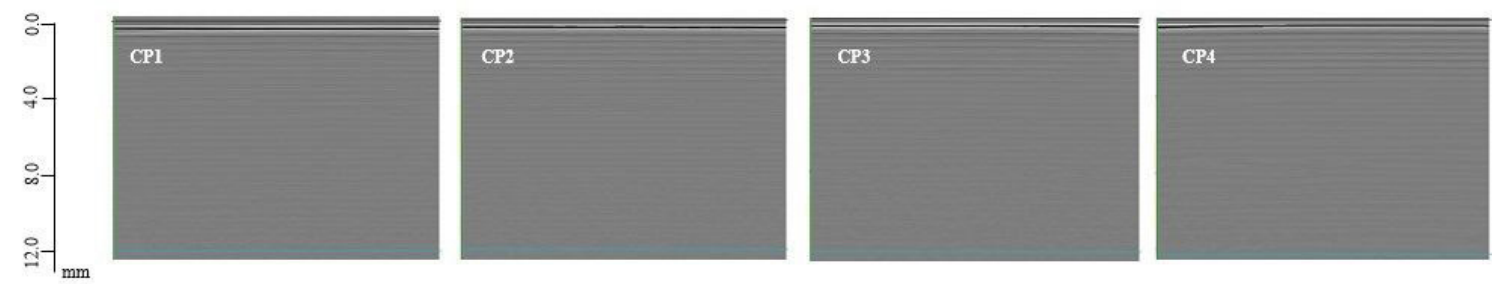

Figure 7. B-Scan images of the samples without defects - Group CP. Scale shows the depths along direction z.
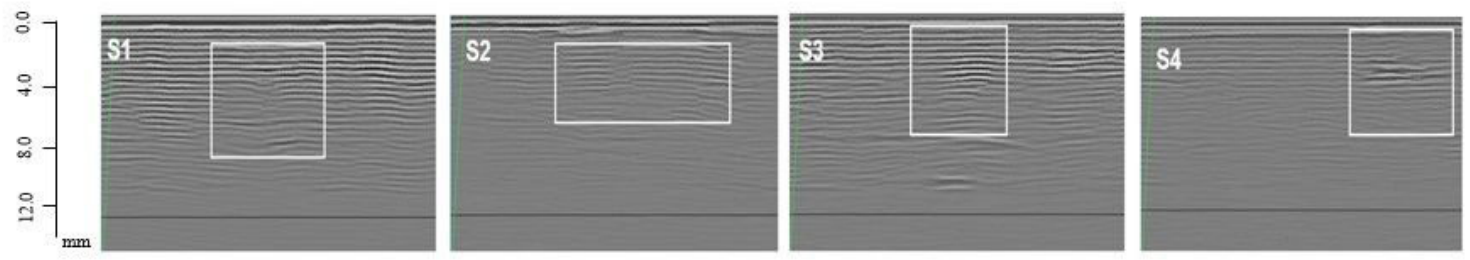

Figure 8. B-Scan images of the samples with fiber waviness - Group S. Scale shows the depths along direction z.
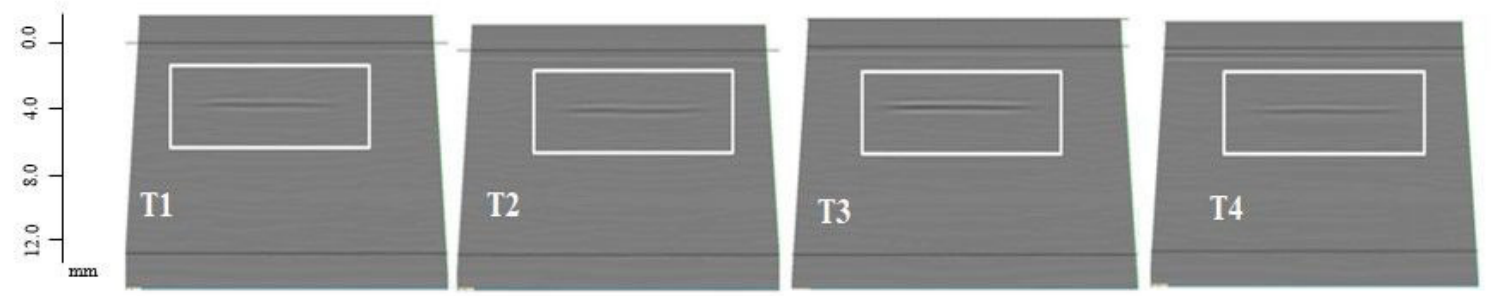

Figure 9. B-Scan image of the samples with delamination - Group T. Scale shows the depths along direction z.

for sample 2 (S2), which was more difficult to identify by B-Scan method.

Figure 11 presents the results for the samples with delamination. In that figure, it was possible to identify the defect through variations of SNR, although it is not so easy for sample 3 (T3). In this group, the difference between maximum and minimum SNR measured was of $1.47 \mathrm{~dB}$.

The propagation of the wave using the PA system occurs by pulse echo. However, the dimensions of surface of the delamination and fiber waviness are different, resulting in 

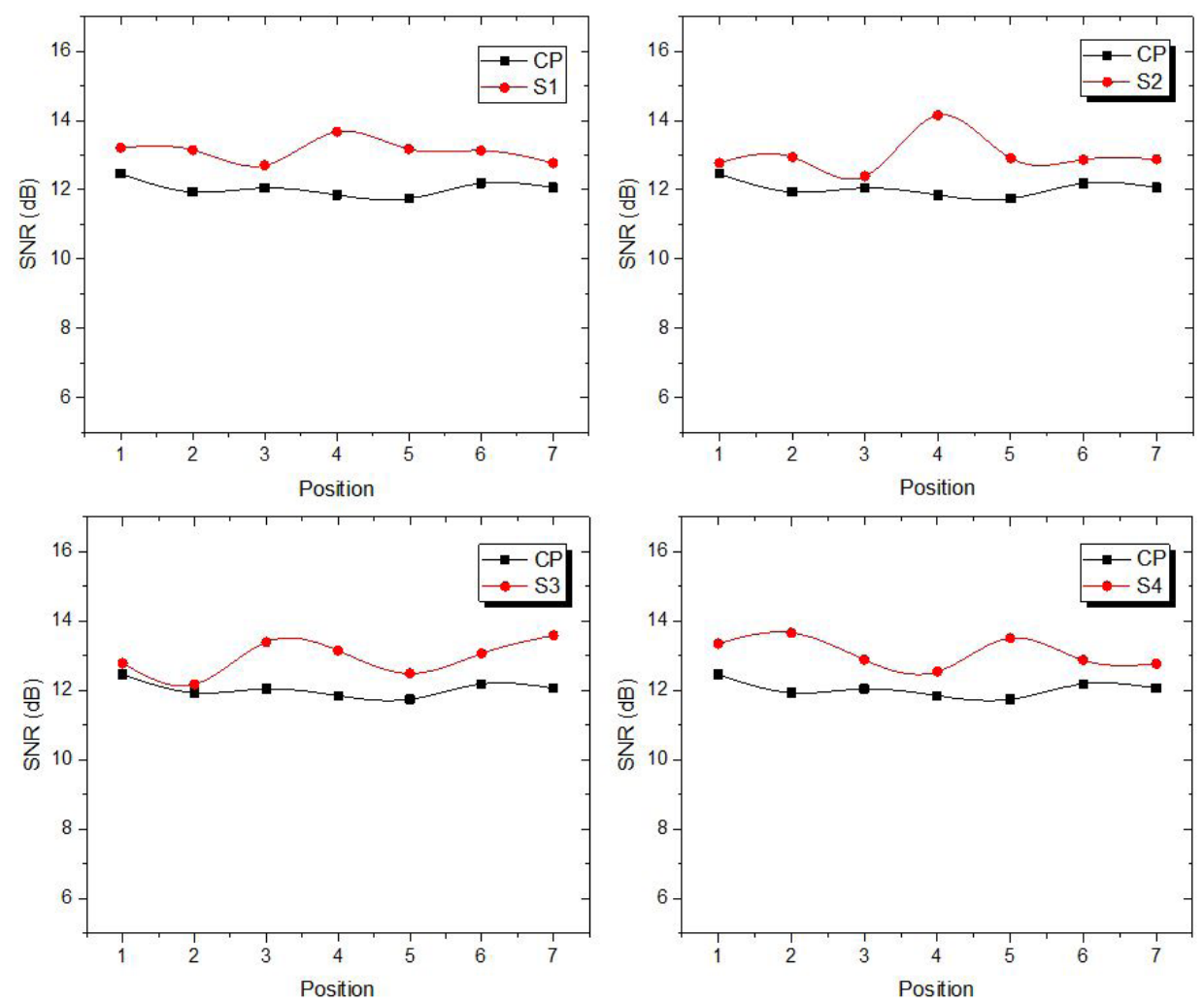

Figure 10. Results of the SNR for samples with fiber waviness.
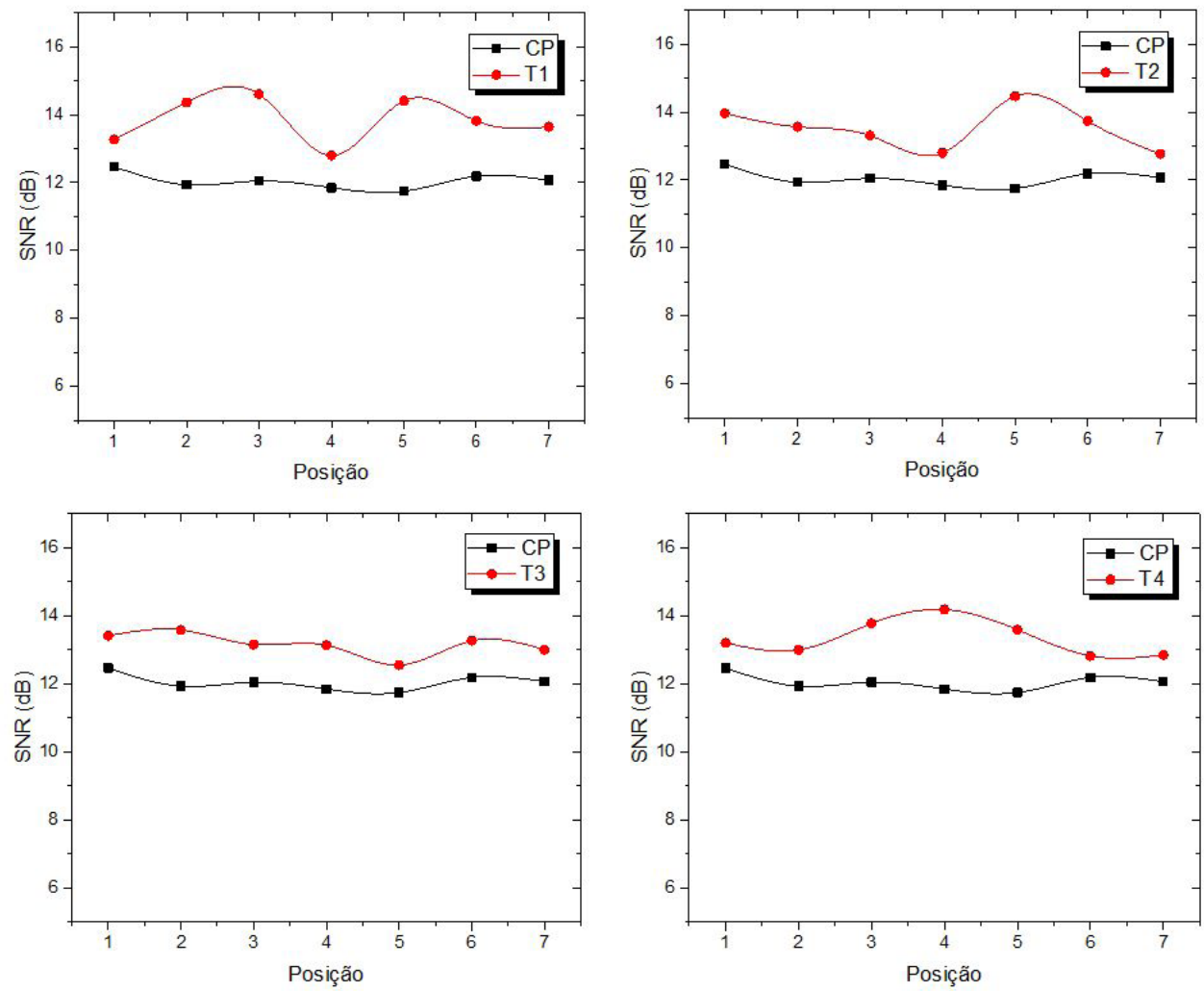

Figure 11. Results of the SNR for samples with delamination. 
a variation of reflections and scattering of the wave when comparing the defects ${ }^{56,57}$. The smaller area and curved surface of fiber waviness generate more scattering, which is better captured with the FMC method and shows better results in the analysis by SNR than by B-Scan image.

The SNR variation allowed locating the region with defect and this position is the same where the defect was detected by B-Scan method; however, only the latter can indicate the depth of the defect. In this way, the methods are complementary.

\section{Conclusions}

This work aimed to evaluate different ultrasonic methods for defect detection in unidirectional carbon/epoxy composite. Defects such as delamination and fiber waviness were investigated using LCR waves through the time-of-flight (TOF), phased array system through B-Scan image, and the SNR variation obtained from the TFM image. The defect of delamination was obtained inserting a piece of Teflon in the sample and the fiber waviness with a sphere of silica.

Using LCR wave, the variation of the time between positions has an average deviation of $0.010 \mu \mathrm{s}$. This variation does not allow for the identification of the presence and location of the defect.

For phased array system with B-Scan, images in real time were obtained in the region with defects, showing that the discontinuity can be identified by the method. The images of samples with delamination clearly determine the presence of the defect and indicate its depth. In samples with fiber waviness, the detection with B-Scan image were not so easy. In three of four samples the visualization of the defect was possible, but without precisely indicating its position.

In the analysis by SNR between positions of the samples, the results show that the variation is greater in samples with defects compared to samples without defects. A difference between maximum and minimum values of SNR of $1.47 \mathrm{~dB}$ was obtained in samples with delamination and $1.31 \mathrm{~dB}$ in samples with fiber waviness, compared to $0.72 \mathrm{~dB}$ in samples without defects.

The results showed that the SNR of the TFM image identified both defects under analysis; the B-Scan image detects the location of the delamination with better accuracy; and that the LCR wave is not efficient to detect these two types of defects in FRP composites.

\section{Acknowledgments}

The authors are grateful to Alltec-Materiais Compositos for providing of the carbon/epoxy samples, National Council for Scientific and Technological Development - CNPq, for financial support (Process 315304/2018-9 and 140771/2016-6) and Coordination of Higher Level Personnel Improvement - CAPES (grant: 88887.499792/2020-00).

\section{References}

1. Soutis C. Recent advances in building with composites. Plast Rubber Compos. 2009;38(9-10):359-66.

2. Shokrieh MM, Mohammadi ARG. The importance of measuring residual stresses in composite materials. In: Shokrieh MM, editor. Residual stresses in composite materials. Duxford: Elsevier; 2014. p. 3-14.

3. Ghasemi AR, Tabatabaeian A, Moradi M. Residual stress and failure analyses of polymer matrix composites considering thermal cycling and temperature effects based on classical laminate plate theory. J Compos Mater. 2019;53(21):3021-32.

4. Duchene P, Chaki S, Ayadi A, Krawczak P. A review of nondestructive techniques used for mechanical damage assessment in polymer composites. J Mater Sci. 2018;53(11):7915-38.

5. Chawla KK. Composite materials. New York: Springer; 2012.

6. Gay D. Composite materials: design and applications. Boca Raton: CRC Press; 2014.

7. Backe D, Balle F. Ultrasonic fatigue and microstructural characterization of carbon fiber fabric reinforced polyphenylene sulfide in the very high cycle fatigue regime. Compos Sci Technol. 2016;126:115-21.

8. Camanho PP, Arteiro A. Analysis models for polymer composites across different length scales. In: Beaumont PWR, Soutis C, Hodzic A, editors. The structural integrity of carbon fiber composites. Cham: Springer International Publishing; 2017. p. 199-279.

9. Meo M, Polimeno U, Zumpano G. Detecting damage in composite material using nonlinear elastic wave spectroscopy methods. Appl Compos Mater. 2008;15(3):115-26.

10. Polimeno U, Meo M. Detecting barely visible impact damage detection on aircraft composites structures. Compos Struct. 2009;91(4):398-402.

11. Franco LALI, Botelho EC, Cândido GM, Rezende MC. Análise fractográfica do modo de falha de compósitos carbono/epóxi. Matéria. 2009;14(1):694-704.

12. Rezende MC. Fractografia de compósitos estruturais. Polímeros. 2007;17(3):E4-11.

13. Kulkarni P, Mali KD, Singh S. An overview of the formation of fibre waviness and its effect on the mechanical performance of fibre reinforced polymer composites. Compos, Part AAppl Sci Manuf. 2020;137:106013.

14. Stecenko T, Piggott MR. Fiber waviness and other mesostructures in filament wound materials. J Reinf Plast Compos. 1997;16(18):1659-74.

15. Baran I, Straumit I, Shishkina O, Lomov SV. X-ray computed tomography characterization of manufacturing induced defects in a glass/polyester pultruded profile. Compos Struct. 2018;195:74-82.

16. Wu C, Gu Y, Luo L, Xu P, Wang S, Li M, et al. Influences of in-plane and out-of-plane fiber waviness on mechanical properties of carbon fiber composite laminate. J Reinf Plast Compos. 2018;37(13):877-91.

17. Chan WS, Wang JS. Influence of fiber waviness on the structural response of composite laminates. Journal Thermoplastic Composite Materials. 1994;7(3):243-60.

18. Karami G, Garnich M. Micromechanical study of thermoelastic behavior of composites with periodic fiber waviness. Compos, Part B Eng. 2005;36(3):241-8.

19. O'Hare Adams D, Hyer MW. Effects of layer waviness on the compression strength of thermoplastic composite laminates. J Reinf Plast Compos. 1993;12(4):414-29.

20. Hörrmann S, Adumitroaie A, Viechtbauer C, Schagerl M. The effect of fiber waviness on the fatigue life of CFRP materials. Int J Fatigue. 2016;90:139-47.

21. Wang J, Potter KD, Etches J. Experimental investigation and characterisation techniques of compressive fatigue failure of composites with fibre waviness at ply drops. Compos Struct. 2013;100:398-403.

22. Sridharan S, editor. Delamination behaviour of composites. Cambridge: Woodhead Publishing Series; 2008.

23. Kaleel I, Carrera E, Petrolo M. Progressive delamination of laminated composites via 1D models. Compos Struct. 2020;235:111799. 
24. Imran $\mathrm{M}$, Khan $\mathrm{R}$, Badshah $\mathrm{S}$. Investigating the effect of delamination size, stacking sequences and boundary conditions on the vibration properties of carbon fiber reinforced polymer composite. Mater Res. 2019;22(2):e20190478.

25. Moura MFSF, Gonçalves JPM, Marques AT, Castro PMST, Prediction of compressive strength of carbon-epoxy laminates containing delamination by using a mixed-mode damage model. Compos Struct. 2000;50(2):151-7.

26. Zeng Z, Tian Q, Wang H, Jiao S, Li J. Testing of delamination in multidirectional carbon fiber reinforced polymer laminates using the vertical eddy current method. Compos Struct. 2019;208:314-21.

27. Mizukami K, Ibrahim ASB, Ogi K, Matvieieva N, Kharabet L, Schulze M, et al. Enhancement of sensitivity to delamination in eddy current testing of carbon fiber composites by varying probe geometry. Compos Struct. 2019;226:111227.

28. Ghadermazi K, Khozeimeh MA, Taheri-Behrooz F, Safizadeh MS. Delamination detection in glass-epoxy composites using step-phase thermography (SPT). Infrared Phys Technol. 2015;72:204-9.

29. Chakrapani SK, Barnard D, Dayal V. Detection of in-plane fiber waviness in composite laminates using guided Lamb modes. AIP Conf Proc. 2014;1581:1134-40.

30. Anastasi RF. Investigation of fiber waviness in a thick glass composite beam using THz NDE. In: SPIE Smart Structures and Materials + Nondestructive Evaluation and Health Monitoring; 2008; San Diego, California. Proceedings. Bellingham: SPIE; 2008. (vol. 6934).

31. Dong J, Kim B, Locquet A, McKeon P, Declercq N, Citrin DS. Nondestructive evaluation of forced delamination in glass fiber-reinforced composites by terahertz and ultrasonic waves. Compos, Part B Eng. 2015;79:667-75.

32. Kessler SS, Spearing SM, Soutis C. Damage detection in composite materials using Lamb wave methods. Smart Mater Struct. 2002;11(2):269-78.

33. Masserey B, Fromme P. Surface defect detection in stiffened plate structures using Rayleigh-like waves. NDT Int. 2009;42(6):56472.

34. Castellano A, Foti P, Fraddosio A, Marzano S, Piccioni MD. Ultrasonic immersion tests for mechanical characterization of multilayered anisotropic materials. In: 2014 IEEE Workshop on Environmental, Energy, and Structural Monitoring Systems Proceedings; 2014; Naples, Italy. Proceedings. New York: IEEE; 2014. p. 1-6.

35. Castellano A, Foti P, Fraddosio A, Marzano S, Piccioni MD. Mechanical characterization of CFRP composites by ultrasonic immersion tests: experimental and numerical approaches. Compos, Part B Eng. 2014;66:299-310.

36. Shoukroun D, Massimi L, Iacoviello F, Endrizzi M, Bate D, Olivo A, et al. Enhanced composite plate impact damage detection and characterisation using X-Ray refraction and scattering contrast combined with ultrasonic imaging. Compos, Part B Eng. 2020;181:107579.

37. Meola C, Boccardi S, Carlomagno GM, Boffa ND, Monaco E, Ricci F. Nondestructive evaluation of carbon fibre reinforced composites with infrared thermography and ultrasonics. Compos Struct. 2015;134:845-53.

38. Yu X, Ratassepp M, Fan Z. Damage detection in quasi-isotropic composite bends using ultrasonic feature guided waves. Compos Sci Technol. 2017;141:120-9.
39. Drinkwater BW, Wilcox PD. Ultrasonic arrays for non-destructive evaluation: a review. NDT Int. 2006;39(7):525-41.

40. Li C, Pain D, Wilcox PD, Drinkwater BW. Imaging composite material using ultrasonic arrays. NDT Int. 2013;53:8-17.

41. Feng B, Ribeiro AL, Ramos HG. Interaction of Lamb waves with the edges of a delamination in CFRP composites and a reference-free localization method for delamination. Measurement. 2018;122:424-31.

42. Zardan JP, Gueudré C, Corneloup G. Study of induced ultrasonic deviation for the detection and identification of ply waviness in carbon fibre reinforced polymer. NDT Int. 2013;56:1-9.

43. Wooh SC, Daniel IM. Wave propagation in composite materials with fibre waviness. Ultrasonics. 1995;33(1):3-10.

44. Zhao G, Wang B, Wang T, Hao W, Luo Y. Detection and monitoring of delamination in composite laminates using ultrasonic guided wave. Compos Struct. 2019;225:111161.

45. Kappatos V, Asfis G, Salonitis K, Tzitzilonis V, Avdelidis NP, Cheilakou E, et al. Theoretical assessment of different ultrasonic configurations for delamination defects detection in composite components. Procedia CIRP. 2017;59:29-34.

46. Zhang Z, Liu M, Li Q, Ang Y. Visualized characterization of diversified defects in thick aerospace composites using ultrasonic B-scan. Composites Communications. 2020;22:100435.

47. Larrañaga-Valsero B, Smith RA, Tayong RB, Fernández-López A, Güemes A. Wrinkle measurement in glass-carbon hybrid laminates comparing ultrasonic techniques: a case study. Compos, Part A Appl Sci Manuf. 2018;114:225-40.

48. Liu M, Xiao H, Hu Q, Zhang Z, Leung CM. Floquet wave theorybased time-corrected ultrasonic total focusing method for fiberreinforced composite laminate. Ultrasonics. 2021;116:106467.

49. Achenbach JD. Wave propagation in elastic solids. Amsterdam: Elsevier; 1973.

50. Bray DE, Stanley RK. Nondestructive evaluation: a tool in design, manufacturing and service. 1st ed. Boca Raton: CRC Press, 1996.

51. Rose JL. Ultrasonic guided waves in solid media. New York: Cambridge University Press; 2014.

52. Chaki S, Ke W, Demouveau H. Numerical and experimental analysis of the critically refracted longitudinal beam. Ultrasonics. 2013;53(1):65-9.

53. Ashizawa T, Mizutani Y, Toyama N, Todoroki A, Suzuki Y. Numerical analysis and experimental observation of ultrasonic wave propagation in CFRP with curved fibers. Compos Struct. 2018;194:316-27.

54. Balasubramaniam K, Whitney SC. Ultrasonic through-transmission characterization of thick fibre-reinforced composites. NDT Int. 1996;29(4):225-36.

55. Wooh S-C, Wang J. Nondestructive characterization of defects using a novel hybrid ultrasonic array sensor. NDT Int. 2002;35(3):155-63.

56. Chang J, Zheng C, Ni Q-Q. NI Q-Q. The ultrasonic wave propagation in composite material and its characteristic evaluation. Compos Struct. 2006;75(1-4):451-6.

57. Zhang Z, Guo S, Li Q, Cui F, Malcolm AA, Su Z, et al. Ultrasonic detection and characterization of delamination and rich resin in thick composites with waviness. Compos Sci Technol. 2020;189:108016. 